\title{
A Glance at the Relationship between Blood Chamber and Chong Mai (Penetrating Vessel)
}

\author{
Tong Zheng Hong* \\ Department of Health Sciences, Taiwan \\ *Corresponding author: Tong Zheng Hong, Department of Health Sciences, Taiwan
}

\section{Introduction}

The primary purpose of the meridians is regulation of the flow Qi and the transportation of Blood throughout the body. The term Jing-mai (經-脈 in Chinese), accurately referring to two words channel (Jing, 經] ) and pulse (mai, 脆), translated into meridian or meridian In English refers in a whole to the connecting and collective system in the body, which is the key to the diagnosis and treatment in acupuncture and TCM. There are a total of the seventytwo major Jing-mai, which are divided into the twelve major meridians considered to be the most important in acupuncture. The main meridian categories are:

a. Twelve Major Meridians connect to the Zang-Fu organs and are the main pathways for transporting qi and Blood throughout the body.

b. Eight Extraordinary Vessels do not pertain to the specific meridian to connect a Zang organ, but connect the twelve primary meridians named Conception Vessel, Governor Vessel, Chong Mai (Thoroughfare/Penetrating Vessel), Belt Vessel, Link Vessels and Heel Vessels.

c. Twelve Divergent or Distinct Meridians are used to connect the yuan (prenatal) and wei (defensive) qi.

The term Xue-shi (血室, Blood Chamber) was first presented discussed by Zhang Zhongjing, the author of Shang-han-lun ( 傷寒論, On Cold Damage), in Jingui Yaolue (金匱要略, Essential Prescriptions of the Golden Coffer). Among the Extraordinary meridians, Chong Mai (Penetrating Vessel) deserves attention with the relationship between Blood Chamber and the functions of Chong Mai.

\section{Chong Mai and blood chamber}

Chong Mai, also called the Sea of Blood, to some extents seems to be exclusive for women with its route and the connections with
Zang-Fu organs for the reproductive system. This vessel originates in the Mingmen area between Zang organs Kidney, ascends through the Uterus to REN1, and emerges with ST30. It goes upwards along the Kidney meridian along the umbilicus, ascends to reach the chest, and finally surround the lips [1]. In the $38^{\text {th }}$ Chapter of the Volume Ling Shu, it describes the main functions of Chong Vessel (Penetrating Vessel) include

a. Connections of Pre- and Postnatal Jing and Qi

b. The regulations of Qi and Blood in the Uterus for the menstrual cycle to facilitate fertility and conception

c. Warming Mingmen to nourish Liver, Kidney, and Spleen

d. Receiving from the Kidney meridian Jing and assistance [1]. In clinical use, this vessel is frequently selected for the route connecting genitals, abdomen, Zang organ Heart, thorax, inner thigh, and the systems of reproduction, digestion and respiration to treat Blood-related menstrual and reproductive issues, hiccups, heart pain, SOB, asthma, and depression. Chong Mai, as discussed above, goes through the Uterus, which is only seen in the women. However; this vessel also passes through the same location in men. The present literature about Chong Mai seems to suggest that it is mostly responsible for the women's health issues. Chong Mai, however, also exists in the men, which arouses the controversy whether or not the functions of this vessel can also be applied to men. The notion Heat penetrating into Blood Chamber is first presented and discussed by Zhang Zhongjing in the Article 10 of Chapter 22 of Jingui Yaolue (金贀要略, Essential Prescriptions of the Golden Coffer) and Article 144 of Shang-han-lun (傷寒論 , On Cold Damage). It says the symptoms of alternating cold and heat occurs with the evil Qi Cold invaded for 7 or 8 days indicates that Heat has penetrated into Blood Chamber. A woman who has this symptom suffers from the Blood stasis, which can be treated 
with Xiao-chai-hu Tang (小柴胡湯, Minor Bupleurum Decoction). It has been argued for long about the definition of Blood Chamber. Zang Jingyue, the author of The Complete Works of Jing Yue ( 景岳全書, Jing Yue Quan Shu) refers it to Uterus since Uterus can be only seen in the women for the menstrual and reproductive functions. However, it is argued by Wang Bin (王冰) in the Tang Dynasty that Blood Chamber is Chong Mai that is the Sea of Blood responsible for women to nourish Blood for meeting with the meridians. The author of Shang-han-lun-zhu (傷寒論注, Note for On Cold Damage) Ker Chin (柯琴) stated Blood Chamber means Liver and Blood stasis can only occurs in women because Blood is stored in Liver [2].

\section{Discussion}

Judging from the actions and components of Xiao-chai-hu Tang (Chai Hu, Huang Qin, Ren Shen, Ban Xia, Sheng Jiang, Zhi Gan Cao, and Da Zao) used to harmonize Shaoyang Stage disorders and tonify the Middle Jiao resulted from the Liver qi stagnation, it is suggested in this study that Blood Chamber in the TCM refers to the symptoms in women and men, in addition to Uterus. The meridian theory indicates that Shaoyang is between Taiyang and Yangming, and the Shayoang syndromes like alternating chills and fever, bitter taste in the mouth, digestive issues of bloating, poor appetite, nausea, stomachache, and vomiting, and pain or discomfort in the costal or hypochondriac region represent that the pathogen is between Internal and External [3]. In addition, all the symptoms can be seen in the men, which suggests Heat cannot only affect women in Blood Chamber but affect men. Chai Hu (柴胡, Radix Bupleuri) is one of the frequently used traditional Chinese medical herbs in treating the Liver-related diseases. Chaihu, derived from the dried roots of Pei (北柴胡, Chaihu Bupleurum Chinense DC.) and Nan Chaihu (南柴胡, Bupleurum scorzonerifolium Willd) [4], is the main component of the most famous and frequently used preparations
Xiao-chai-hu Tang (decoction) and Da-chai- hu Tang (decoction). The major action of Bupleurum chinense DC. is dispersing stagnated Liver qi while raising Yang Qi in the Middle Jiao deficiency pattern is the action of Bupleurum scorzonerifolium Willd. On the other hand, Uterus, nourished by Blood, is the residence for the fetus. One of the functions of Liver is to store Blood that can ensure and promote Qi to flow freely within the body. Blood stasis occurs with Liver qi stagnation which indicates the auto nerve system malfunctions resulting in dysmenorrhea and depression [5]. In other words, Heat in the Blood Chamber is the result of stagnated Liver qi in particular since Xiao-chai-hu Tang can be selected for the treatment of this symptom.

\section{Conclusion}

The discussions of Blood Chamber show that each term or concept in the TCM cannot be fixed because TCM develops with the inductive logic thinking, which emphasizes the similarity. One of the reasons is that lexical interpretations vary with the changes of time. Scientific evidence is needed in the future to explain the symptoms biologically and explore the underling mechanisms of the meridians.

\section{References}

1. Szmelskyj I, Aquilina L, Szmelskyj A (2015) Acupuncture for IVF and assisted reproduction: An integrated approach to treatment and management. Churchhill Livingstone, UK.

2. KKnews. What are conception vessel, governor vessel, Chong Mai?

3. Sacred Lotus Chinese medicine. Differentiation of Syndromes According to the Six Stages.

4. Hong TZ (2018) Challenges in learning and understanding traditional Chinese medicine and acupuncture. Open Acc J Comp \& Alt Med 1(1): 5-9.

5. Hong TZ (2018) Understanding the TCM role of liver in the treatment of cough. LOJ Phar \& Cli Res 1(2): 38-40.

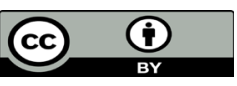

This work is licensed under Creative Commons Attribution 4.0 License

To Submit Your Article Click Here: Submit Article

DOI: 10.32474/OAJCAM.2019.01.000117

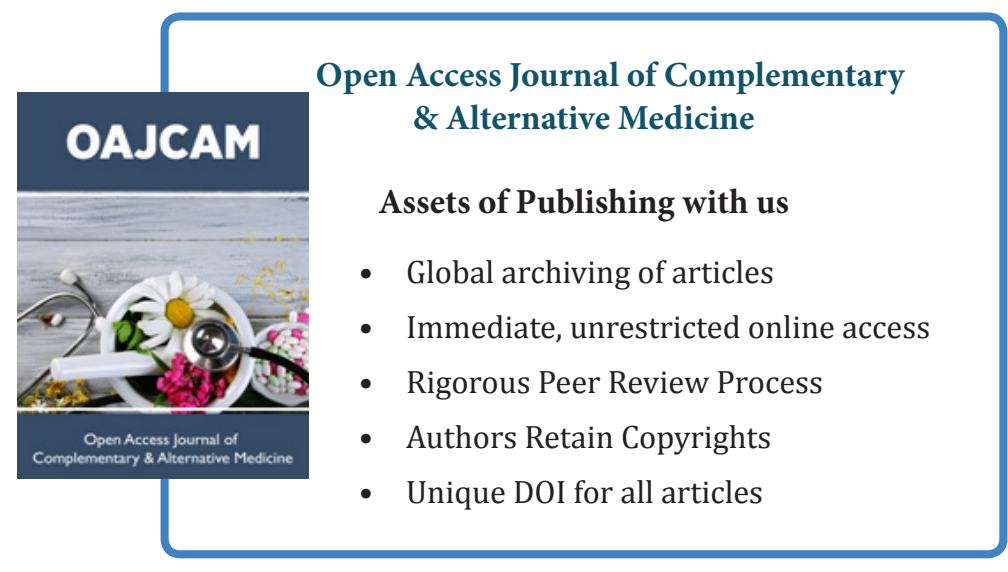

